\title{
Assembléias de peixes associados às macrófitas aquáticas em ambientes alagáveis do Pantanal de Poconé, MT, Brasil
}

\author{
Valéria Milani $^{1,3}$, Francisco de Arruda Machado ${ }^{2}$ \& Veviane Cristina Ferreira e Silva ${ }^{1}$ \\ ${ }^{1}$ Programa de Pós-graduação em Ecologia e Conservação da Biodiversidade, \\ Universidade Federal de Mato Grosso - UFMT, CEP 78060-900, Cuiabá, MT, Brasil, \\ e-mail: veviane.cristina@hotmail.com \\ ${ }^{2}$ Departamento de Biologia, Instituto de Biociências, Universidade Federal de Mato Grosso - UFMT, \\ CEP 78060-900,Cuiabá, MT, Brasil,e-mail:salaminus@ufmt.br \\ ${ }^{3}$ Autor para correspondência: Valéria Milani, e-mail: valeriamilaninx@yahoo.com.br
}

MILANI, V., MACHADO, F.A. \& SILVA, V.C.F. Fish assemblages associated to aquatic macrophytes in wetland environments of Pantanal de Poconé, State of Mato Grosso, Brazil. Biota Neotrop. 10(2): http:// www.biotaneotropica.org.br/v10n2/en/abstract?inventory+bn02310022010.

\begin{abstract}
We examined the composition, richness and biomass of fish assemblages associated with aquatic macrophytes in temporary environments of the Pantanal de Poconé, State of Mato Grosso, Brazil. The fish were captured in April 2008 in five loan boxes and five locations along Coqueiro Bay, using seine nets at three points within each site. A total of 9646 specimens were captured, distributed in seven orders, 17 families and 59 species. The fish community was composed mainly of small-sized species (Characidae) and some juveniles of medium-sized species (Erythrinidae and Gymnotidae). In this study, few species were very abundant, some showed intermediate abundance, and most had low abundance. According to an analysis of similarity (Anosim) there was no difference in species composition between the environments. Species richness, total abundance and biomass did not vary spatially. Thus, the results suggest that the similarity of the fish fauna can be attributed to flooding, since this phenomenon homogenizes the abiotic characteristics and enlarges the area for the distribution of fish. This fact seems to be very important for these small fish species, since many species need to inhabit specific sites during certain stages; for example, these environments may function as temporary refuges. This aspect may ensure the survival of many individuals until they reach a stage in life where predation is significantly reduced.
\end{abstract}

Keywords: temporary habitats, species composition, ichthyofauna, similarity, flooding.

MILANI, V., MACHADO, F.A. \& SILVA, V.C.F. Assembléias de peixes associados às macrófitas aquáticas em ambientes alagáveis do Pantanal de Poconé, MT, Brasil. Biota Neotrop. 10(2): http://www.biotaneotropica. org.br/v10n2/pt/abstract?inventory+bn02310022010.

Resumo: Este estudo avaliou a composição, riqueza e a biomassa das assembléias de peixes associadas às macrófitas aquáticas em ambientes temporários do Pantanal de Poconé - MT. A coleta foi realizada em abril de 2008, em cinco caixas de empréstimo e cinco locais na baía do Coqueiro, por meio de rede de cerco, em três pontos dentro de cada local. Foram capturados 9646 exemplares, distribuídos em sete ordens, 17 famílias e 59 espécies. A comunidade de peixes foi composta principalmente por espécies de pequeno porte (Characidae) e alguns juvenis de espécies de médio porte (Erythrinidae e Gymnotidae). Poucas espécies foram muito abundantes, algumas apresentaram abundância intermediária e a maioria apresentou baixa abundância. A análise de similaridade (Anosim) mostrou que não existe diferença na composição de espécies entre os ambientes. A riqueza de espécie e a biomassa dos peixes também não diferiram espacialmente. Desta forma, nossos resultados sugerem que a similaridade da ictiofauna é uma conseqüência da inundação, uma vez que esta permite a homogeneização das características abióticas e amplia a área de distribuição dos peixes. Este fato aparenta ser de grande importância para estes pequenos peixes, considerando que muitas espécies necessitam de lugares específicos em determinados estágios, como, por exemplo, esses ambientes temporários que podem servir de refúgio. Este aspecto pode garantir a sobrevivência de muitos indivíduos até atingirem uma fase da vida em que a predação sobre si diminui consideravelmente.

Palavras-chave: hábitats temporários, composição de espécies, ictiofauna, similaridade, inundação. 


\section{Introdução}

A composição da ictiofauna local pode ser descrita como resultado dos aspectos físicos do ambiente, tais como heterogeneidade do hábitat e gradientes físico-químicos (Ricklefs 1987, Jackson et al. 2001), combinados à probabilidade de colonização e extinção de cada espécie (Pazim et al. 2006), o que justifica as especializações das espécies de peixes para um determinado ambiente físico que possa garantir sua sobrevivência (Moran-Lopez et al. 2005).

Os processos ecológicos no sistema Rio Paraguai e a planície inundável são regulados pelo pulso de inundação (Junk et al. 1989), que sazonalmente muda a estrutura dos canais e dos pequenos lagos, tornando a planície de inundação alternadamente adequada para organismos aquáticos e terrestres (Junk et al. 2006). Essa mudança no nível da água faz com que a população de peixes tenha seus ciclos de vida relacionados a flutuações no nível dos rios (Junk et al. 1989). No período chuvoso, os altos níveis da água resultam na expansão lateral dos corpos de água permanentes através da planície de inundação, aumentando a conectividade e formando novos corpos de água temporários, disponibilizando novos hábitats para organismos aquáticos (Petry et al. 2003a). Nessa época, há uma proliferação extraordinária de vegetação aquática, principalmente nas margens desses ambientes, nas quais concentram bancos de macrófitas aquáticas, que são utilizados por uma gama de organismos, e são consideradas de grande importância para a manutenção dos estoques pesqueiros em planícies inundáveis (Oliveira et al. 2006). Desta forma, a inundação, além de proporcionar uma alta diversidade de hábitats, aumenta também os tipos especiais de hábitats (Robinson et al. 2002).

Com a conexão dos lagos e rios no período de inundação, o potencial de alterações na estrutura das assembléias aumenta, pois ovos, larvas e peixes adultos podem sofrer reorganização espacial entre os corpos de água (Lowe-MCconnell 1987). Os peixes deixam seus ambientes para selecionarem locais de desova, hábitats que reduzem a sua probabilidade ou da sua prole de ocuparem um lago desfavorável durante a isolação (Rodríguez \& Lewis 1994, Saint-Paul et al. 2000) e essa movimentação pode fazer com que ambientes distintos, dentro da planície inundável, tenham faunas similares durante o período chuvoso (Thomaz et al. 2007).

Estudos sobre corpos de água temporários, apesar de enfatizar a aparência árdua do ambiente, devido a alterações entre a fase seca e a chuvosa, reconhecem que estes oferecem uma variedade de oportunidades que tem sido explorada por diferentes espécies (Collinson et al. 1995), entretanto, são pouco estudados por possuírem comunidades dominadas por espécies de pequeno porte, sem interesse comercial ou de pesca recreativa (Eggleton et al. 2005). No Pantanal de Poconé, além dos corpos de água permanentes de maior porte chamados de baías, existem depressões provenientes de ações antrópicas, as quais são denominadas caixas de empréstimo, que são formadas durante a construção de rodovias, que, periodicamente, enchem-se de água, formando pequenas lagoas, ao lado das margens da estrada, povoadas por muitos organismos aquáticos. Apesar de serem ambientes comuns nessa região do Pantanal, há pouca informação, em termos quantitativos, sobre a comunidade de peixes que os habita. Assim, determinar a importância desses ambientes para determinados grupos de organismos pode proporcionar entendimentos ecológicos básicos sobre esses locais e as suas comunidades. Neste estudo verificamos quais espécies de peixes colonizam esses ambientes temporários e se a composição de espécies, riqueza e biomassa das assembléias de peixes associadas às macrófitas aquáticas difere entre esses ambientes inundáveis no Pantanal de Poconé.

\section{Material e Métodos}

\section{1. Área de estudo}

$\mathrm{Na}$ área de estudo predomina o clima quente, com pronunciada estação seca de maio a setembro e estação chuvosa de outubro a abril. A pluviosidade anual é de $1.250 \mathrm{~mm}$ na parte norte (Cáceres) e de $1.089 \mathrm{~mm}$ na parte sul (Corumbá) e a temperatura média, próximo a Cuiabá, é de $27,4{ }^{\circ} \mathrm{C}$ em dezembro e $21,4^{\circ} \mathrm{C}$ em julho (Junk et al. 2006). O padrão de inundação varia entre os diferentes pantanais, podendo ser pelo transbordamento dos rios ou pela chuva local (Hamilton et al. 2002).

O estudo foi conduzido em caixas de empréstimo e na baía do Coqueiro ( $16^{\circ} 23^{\prime} 23^{\prime}$ " S e 56 $16^{\circ}$ ' 30" O), na planície de inundação do Rio Cuiabá, Pantanal de Poconé, Estado de Mato Grosso, Brasil (Figura 1). As caixas de empréstimo são lagoas formadas ao longo de barrancos, onde a terra foi removida para a construção de estradas. Esse movimento de terra deixa desníveis suficientes que permitem o acúmulo de um volume considerável de água durante o período das chuvas, quando estas lagoas se unem formando um imenso canal nas bordas, dos dois lados da estrada. No período da vazante, já é possível identificar essas lagoas separadamente quando algumas secam e outras, onde as escavações foram mais profundas, podem manter a água durante o ano todo. A duração do período seco difere entre as caixas de empréstimo, que são tipicamente rasas, com densa cobertura de macrófitas, sendo Eichhornia azurea (Pontederiaceae) a espécie dominante.

A baía do Coqueiro (16 $26^{\circ} 23^{\prime}$ 'S e $56^{\circ} 17^{\prime} 30^{\prime}$ ' O) está localizada no município de Nossa Senhora do Livramento e é um lago raso, típico de planície de inundação, da área inundada pelo Rio Cuiabá na parte norte do Pantanal (Wantzen et al. 2002). Durante o pico da estação chuvosa (janeiro-abril), a baía do Coqueiro conecta-se com o Rio Piraim, um tributário do Rio Cuiabá (Wantzen et al. 2002). E no período de estudo a vegetação flutuante era formada por uma associação de plantas aquáticas e semiaquáticas, destacando-se entre elas a Pontederia cf. sulbovata, Pontederia cf. rotundifolia e Eichhornia azurea.

\section{Amostragem}

A amostragem foi realizada em abril de 2008, em cinco caixas de empréstimo e cinco pontos na baía do Coqueiro, utilizando rede de cerco (6 $\mathrm{m}$ de comprimento, 1,5 de altura nas extremidades, $3 \mathrm{~m}$ no centro e malha de $2,5 \mathrm{~mm}$ ), que, armada, foi sustentada por duas pessoas, segurando-a pelas extremidades, enquanto uma terceira pessoa cortava as macrófitas presas no substrato e, subseqüentemente, trazida para o barco, onde os peixes foram cuidadosamente separados das plantas. Em cada local foram realizados três lances consecutivos, independentemente do tamanho do banco de macrófita, sendo que este procedimento teve o intuito de obter a melhor representatividade possível da comunidade de peixes. As coletas foram realizadas pela manhã e no crepúsculo vespertino, os peixes foram fixados em solução de formol $10 \%$ e transferidos para álcool a $70 \%$ para conservação. Posteriormente os mesmos foram identificados com auxílio de chaves taxonômicas contidas em Britski et al. (2007) e algumas espécies confirmadas por especialistas. O peso (g) foi obtido por espécies de cada ponto, utilizando balança GEHAKA, Modelo BG:400 (0,01 g de precisão).

A biomassa de macrófitas foi amostrada com o auxílio de um quadrado metálico $\left(0,70 \mathrm{~m}^{2}\right)$, sendo este colocado aleatoriamente em cada local de coleta e toda a vegetação contida nessa área foi removida. Em laboratório, as amostras foram levadas à estufa $\left(60{ }^{\circ} \mathrm{C}\right.$ por 7 dias) para obtenção de seu peso seco. A transparência da água foi medida com disco de Secchi e a profundidade com uma régua graduada. As medidas de oxigênio dissolvido (mg. $\left.\mathrm{L}^{-1}\right), \mathrm{pH}$, 


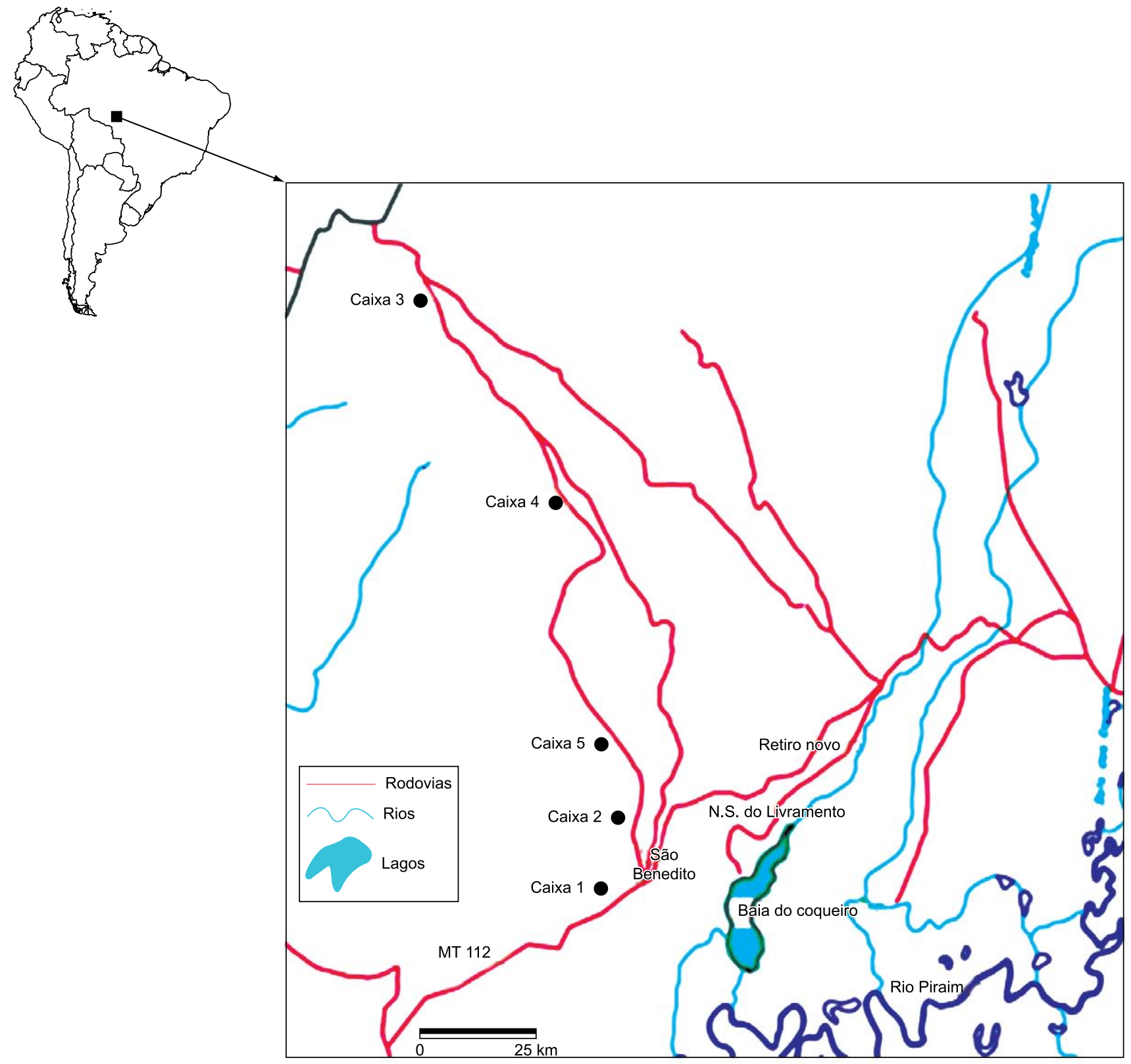

Figura 1. Localização geográfica da área de estudo, município de N. S. do Livramento, Pantanal Mato-Grossense.

Figure 1. Geographical location of the study area at N. S. do Livramento municipality, Pantanal de Poconé, State of Mato Grosso, Brazil.

condutividade $\left(\mu \mathrm{S} . \mathrm{cm}^{-1}\right)$ e temperatura $\left({ }^{\circ} \mathrm{C}\right)$ foram tomadas utilizando uma multisonda YSI modelo 556.

\section{Análise de dados}

Para analisar as diferenças espaciais nas características físicas e químicas, foi usado o teste $t$.

Com o objetivo de verificar o padrão de distribuição de abundância das espécies na comunidade de peixes, utilizamos o rank de abundância. Como a abundâncias das espécies (n) apresentam várias ordens de magnitude, foram transformadas, utilizando log $10(n+1)$ para que pudessem ser visualizadas em um mesmo gráfico (Magurran 2004).

As possíveis diferenças espaciais na riqueza de espécies, na biomassa e no número total de peixes, foram avaliadas utilizado o teste $t$. Com a finalidade de analisar se existe diferença espacial na composição de espécies entre os dois ambientes, foi realizada uma análise de similaridade (ANOSIM), um teste de permutação baseado nas similaridades das amostras (Clarke 1993), e graficamente ilustramos por uma análise de cluster UPGMA. Ambas as análise foram feitas usando dados qualitativos (presença-ausência) e quantitativos (abundância) e foram realizadas utilizando os programas estatísticos SYSTAT (2004), versão 11 e R (R Development Core Team 2005).

\section{Resultados}

\section{Características físicas e químicas}

De acordo com o teste $t$, os dois ambientes diferiram significativamente $(\mathrm{p}<0,05)$ apenas com relação às variáveis biomassa 
de macrófita, $\mathrm{pH}$ e temperatura (Tabela 1), sendo que a média da biomassa de macrófita nas caixas de empréstimo $\left(467,8 \mathrm{~g} \cdot \mathrm{m}^{-2}\right)$ foi superior àquela observada na baía do Coqueiro $\left(118,72\right.$ g.m $\left.{ }^{-2}\right)$. A temperatura da água e o $\mathrm{pH}$ também apresentaram valores superiores nas caixas de empréstimo do que na baía do coqueiro, entretanto, em relação às demais variáveis não houve variação significativa entre os dois ambientes.

Os dois ambientes estudados apresentaram água totalmente transparente, com concentração média de oxigênio na água de 2,36 mg.L $\mathrm{L}^{-1}$ nas caixas de empréstimo e de $1,18 \mathrm{mg} . \mathrm{L}^{-1}$ na baía do Coqueiro. A condutividade da água foi baixa nos dois ambientes, sendo as médias iguais a $20,6 \mu \mathrm{S} . \mathrm{cm}^{-1}$ nas caixas de empréstimo e $13 \mu \mathrm{S} . \mathrm{cm}^{-1}$ na baía do Coqueiro (Tabela 1).

\section{Composição da ictiofauna}

Foram capturados 9.646 exemplares, distribuídos em sete ordens, 17 famílias e 59 espécies (Tabela 2). Characiformes foi a ordem dominante nas caixas de empréstimo (82\%) e na baía do Coqueiro (80\%), seguida por Perciformes (10 e $11 \%$ respectivamente) (Tabela 2). Já Lepdosireniformes ocorreu apenas na baía do Coqueiro e foi a ordem menos abundante neste ambiente $(0,12 \%)$, enquanto nas caixas de empréstimo, Gymnotiformes foi a ordem com abundância relativa mais baixa $(0,09 \%)$.

A ordem com maior número de espécies também foi Characiformes tanto nas caixas de empréstimo (31 espécies), quanto na baía do coqueiro (29 espécies), seguida por Siluriformes (nove espécies) nas caixas de empréstimo e Perciformes (seis espécies) na baía do Coqueiro (Figura 2). Synbranchiformes foi a ordem com o menor número de espécie nas caixas de empréstimo (uma espécie), já na baía do Coqueiro Lepdosireniformes e Synbranchiformes foram as ordens menos representativas (uma espécie cada).

Characidae foi a família mais abundante tanto nas caixas de empréstimo, quanto na baía do Coqueiro, representando 76 e $72 \%$ do total de indivíduos capturados em cada um dos ambientes, respectivamente (Tabela 2). Chiclidae destacou-se como a segunda família com maior abundância relativa nos dois ambientes, com $10 \%$ dos indivíduos coletados nas caixas de empréstimo e $11 \%$ da baía do Coqueiro. As famílias menos abundantes nas caixas de empréstimo foram Sternopygidae, Anostomidae e Gymnotidae, com apenas um indivíduo cada, e na baía do Coqueiro foram Anostomidae e Gymnotidae, também cada uma com um indivíduo.

Poucas espécies foram muito abundantes, algumas apresentaram abundância intermediária e muitas foram pouco abundantes (Figura 3), sendo que $23,7 \%$ das espécies apresentaram abundância superior a 100 indivíduos, 30,5\% apresentaram de 11 a 100 indivíduos e

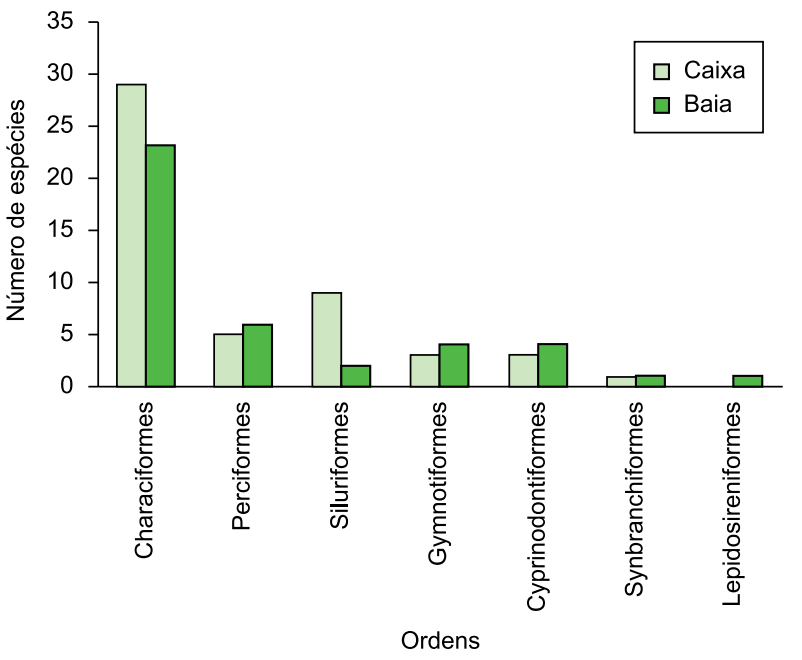

Figura 2. Distribuição da riqueza de espécies agrupadas por ordens taxonômicas na Baía do Coqueiro e nas caixas de empréstimo, Pantanal de Poconé, MT, Brasil.

Figure 2. Species richness distribution grouped by taxonomic order at Coqueiro Bay and in loan boxes, Pantanal de Poconé, State of Mato Grosso, Brazil.

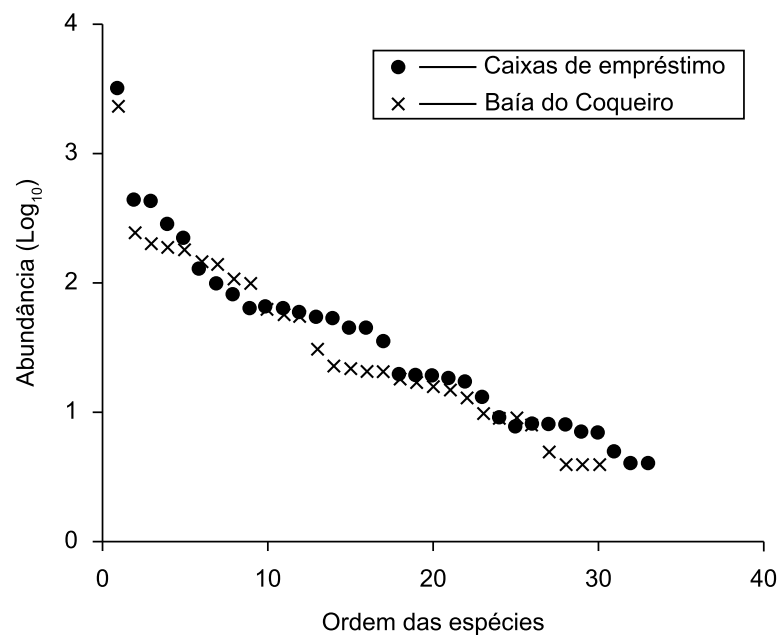

Figura 3. Distribuição de abundâncias da assembleia de peixes das caixas de empréstimo e da baía do Coqueiro, Pantanal de Poconé, MT, Brasil.

Figure 3. Abundance distribution of the fish assemblage in loan boxes and at Coqueiro Bay, Pantanal de Poconé, State of Mato Grosso, Brazil.

Tabela 1. Resumo das características ambientais (média \pm desvio padrão) e resultado do teste $t(\mathrm{~T})$ comparando os locais amostrados no Pantanal de Poconé, MT, Brasil.

Table 1. Summary of the environmental characteristics (mean \pm standard deviation) and results of the test $t$ (T) comparing the sampled sites in Pantanal de Poconé, State of Mato Grosso, Brazil.

\begin{tabular}{|c|c|c|c|c|c|}
\hline Variável & Caixa & Baía & $\mathbf{T}$ & DF & $\mathbf{P}$ valor \\
\hline Oxigênio (mg.L $\left.\mathrm{L}^{-1}\right)$ & $2,362 \pm 1,808$ & $1,18 \pm 0,385$ & $-1,828$ & 8 & 0,105 \\
\hline $\mathrm{pH}$ & $7,020 \pm 0,167$ & $6,59 \pm 0,294$ & $-2,833$ & 8 & $0,022 *$ \\
\hline Condutividade $\left(\mu \mathrm{S} . \mathrm{cm}^{-1}\right)$ & $20,6 \pm 13,107$ & $13 \pm 1$ & $-1,293$ & 8 & 0,232 \\
\hline Temperature $\left({ }^{\circ} \mathrm{C}\right)$ & $31,224 \pm 1,40$ & $29,5 \pm 0,23$ & $-2,807$ & 8 & $0,023 *$ \\
\hline Biomassa de macrófita $\left(\mathrm{g} \cdot \mathrm{m}^{-2}\right)$ & $467,8 \pm 210,9$ & $118,72 \pm 29$ & $-3,667$ & 8 & $0,006^{*}$ \\
\hline Profundidade $(\mathrm{cm})$ & $81,54 \pm 22,45$ & $65,92 \pm 10,52$ & $-1,409$ & 8 & 0,197 \\
\hline Transparência $(\mathrm{cm})$ & Total & Total & - & - & - \\
\hline
\end{tabular}

$\mathrm{DF}=$ graus de liberdade, $\mathrm{p}=$ probabilidade $* \mathrm{p}<0,05$ : diferença significativa.

$\mathrm{DF}=$ degrees of freedom, $\mathrm{p}=$ probability $* \mathrm{p}<0.05$ : significant difference. 
Tabela 2. Abundância de peixes por ordens, famílias e espécies, com número de indivíduos (N), abundância relativa (N\%) e freqüência de ocorrência (F\%) por tipo de ambiente amostrado no Pantanal de Poconé, MT, Brasil.

Table 2. Abundance of fish by order, family and species, with number of individuals (N) and frequency of occurrence (F\%) by type of environment sampled at Pantanal de Poconé, State of Mato Grosso, Brazil.

\begin{tabular}{|c|c|c|c|c|c|c|}
\hline \multirow[t]{2}{*}{ Ordem/Família/Espécies } & \multicolumn{3}{|c|}{ Caixa } & \multicolumn{3}{|c|}{ Baía } \\
\hline & $\mathbf{N}$ & $\mathbf{N \%}$ & F\% & $\mathbf{N}$ & $\mathbf{N \%}$ & F\% \\
\hline Characiformes & 4570 & 82,24 & 100 & 3293 & 80,53 & 100 \\
\hline Characidae & 421 & 7,58 & 100 & 2968 & 72,58 & 100 \\
\hline \multicolumn{7}{|l|}{ Tetragonopterinae } \\
\hline Gymnocorymbus ternetzi Boulenger, 1895 & 64 & 1,15 & 100 & 16 & 0,39 & 40 \\
\hline Markiana nigripinnis Perugia, 1891 & 1 & 0,02 & 20 & - & - & - \\
\hline Moenkhausia dichroura Kner, 1858 & 53 & 0,95 & 80 & 149 & 3,64 & 80 \\
\hline Moenkhausia sanctaefilomenae Steindachner,1907 & 5 & 0,09 & 60 & 2 & 0,05 & 20 \\
\hline Astyanax asuncionensis Gery, 1972 & 1 & 0,02 & 20 & - & - & - \\
\hline Hemigrammus ulreyi Boulenger, 1895 & 7 & 0,13 & 60 & 108 & 2,64 & 80 \\
\hline Psellogrammus kennedyi Eigenmann, 1903 & 63 & 1,13 & 100 & 4 & 0,10 & 20 \\
\hline Hyphessobrycon elachys M. Weitzman, 1985 & 64 & 1,15 & 100 & 57 & 1,39 & 100 \\
\hline Hyphessobrycon eques Steindachner, 1882 & 19 & 0,34 & 60 & 192 & 4,70 & 80 \\
\hline Phenacogaster tegatus Eigenmann, 1911 & 2 & 0,04 & 20 & - & - & - \\
\hline \multicolumn{7}{|l|}{ Aphyocharinae } \\
\hline Megalamphodus megalopterus Eigenmann, 1915 & - & - & - & 1 & 0,02 & 20 \\
\hline Aphyocharax paraguayensis Eigenmann, 1915 & 81 & 1,46 & 100 & 63 & 1,54 & 100 \\
\hline Aphyocharax anisitsi Eigenmann ; Kennedy, 1903 & 131 & 2,36 & 100 & 10 & 0,24 & 80 \\
\hline Aphyocharax rathbuni Eigenmann, 1907 & 58 & 1,04 & 60 & 18 & 0,44 & 80 \\
\hline \multicolumn{7}{|l|}{ Cheirodontinae } \\
\hline Holoshestes pequira Steindachner, 1882 & - & - & - & 9 & 0,22 & 20 \\
\hline Serrapinnus calliurus Boulenger, 1900 & 3193 & 57,46 & 100 & 2326 & 56,88 & 100 \\
\hline Serrapinnus kriegi Schindler, 1937 & 434 & 7,81 & 60 & - & - & - \\
\hline \multicolumn{7}{|l|}{ Characinae } \\
\hline Roeboides bonariensis Steindachner, 1879 & 19 & 0,34 & 20 & - & - & - \\
\hline Roeboides prognathus Boulenger, 1895 & 3 & 0,05 & 20 & - & - & - \\
\hline \multicolumn{7}{|l|}{ Acestrorhynchinae } \\
\hline Acestrorynchus pantaneiro Menezes, 1992 & 8 & 0,14 & 60 & 2 & 0,05 & 40 \\
\hline \multicolumn{7}{|l|}{ Stethaprioninae } \\
\hline Poptella paraguayensis Eigenmann, 1907 & 3 & 0,05 & 20 & 1 & 0,02 & 20 \\
\hline \multicolumn{7}{|l|}{ Myleinae } \\
\hline Metynnis mola Eigenmann \& Kennedy, 1903 & 8 & 0,14 & 80 & 8 & 0,20 & 60 \\
\hline Myloplus levis Eigenmann \& McAtee, 1907 & 1 & 0,02 & 20 & - & - & - \\
\hline \multicolumn{7}{|l|}{ Serrasalminae } \\
\hline Pygocentrus nattereri Kner, 1858 & 1 & 0,02 & 20 & - & - & - \\
\hline Serrasalmus maculatus Kner, 1858 & 2 & 0,04 & 20 & 2 & 0,05 & 40 \\
\hline Crenuchidae & 36 & 0,65 & & 31 & 0,76 & \\
\hline \multicolumn{7}{|l|}{ Characidiinae } \\
\hline Characidium aff. zebra Eigenmann, 1909 & 36 & 0,65 & 80 & 31 & 0,76 & 100 \\
\hline Curimatidae & 74 & 1,33 & 100 & 78 & 1,91 & 80 \\
\hline Cyphocharax gillii Eigenmann \& Kennedy, 1903 & 55 & 0,99 & 60 & 23 & 0,56 & 80 \\
\hline Curimatopsis myersi Vari, 1982 & 19 & 0,34 & 60 & 55 & 1,35 & 40 \\
\hline Anostomidae & 1 & 0,02 & 20 & 1 & 0,02 & 20 \\
\hline Leporinus lacustris Campos, 1945 & 1 & 0,02 & 20 & 1 & 0,02 & 20 \\
\hline Lebiasinidae & 221 & 3,98 & 100 & 202 & 4,94 & 100 \\
\hline
\end{tabular}


Tabela 2. Continuação...

\begin{tabular}{|c|c|c|c|c|c|c|}
\hline \multirow[t]{2}{*}{ Ordem/Família/Espécies } & \multicolumn{3}{|c|}{ Caixa } & \multicolumn{3}{|c|}{ Baía } \\
\hline & $\mathbf{N}$ & $\mathbf{N \%}$ & $\mathbf{F \%}$ & $\mathbf{N}$ & $\mathbf{N \%}$ & F\% \\
\hline \multicolumn{7}{|l|}{ Pyrrhulininae } \\
\hline Pyrrhulina australis Eigenmann \& Kennedy, 1903 & 221 & 3,98 & 100 & 202 & 4,94 & 100 \\
\hline Erythrinidae & 17 & 0,31 & 100 & 13 & 0,32 & 80 \\
\hline Hoplias malabaricus Bloch, 1794 & 17 & 0,31 & 100 & 13 & 0,32 & 80 \\
\hline Siluriformes & 314 & 5,65 & 100 & 19 & 0,46 & 100 \\
\hline Auchinepteridae & 11 & 0,20 & 40 & - & - & \\
\hline Parauchenipterus striatulus Steindachner, 1876 & 7 & 0,13 & 40 & - & - & \\
\hline Entomocorus benjamini Eigenmann, 1917 & 4 & 0,07 & 20 & - & - & - \\
\hline Doradidae & 2 & 0,04 & 20 & - & - & - \\
\hline $\begin{array}{l}\text { Meradoras nheco Higuchi, Birindelli, Sousa \& } \\
\text { Britski, } 2007\end{array}$ & 2 & 0,04 & 20 & - & - & - \\
\hline Callichthyidae & 282 & 5,07 & 100 & 19 & 0,46 & 100 \\
\hline Hoplosternum littorale Hancock, 1828 & 1 & 0,02 & 20 & - & - & - \\
\hline Hoplosternum pectorale Boulenger, 1895 & 0 & 0,00 & 0 & 4 & 0,10 & 20 \\
\hline $\begin{array}{l}\text { Corydoras hastatus Eigenmann \& Eigenmann, } \\
1888\end{array}$ & 281 & 5,06 & 100 & 15 & 0,37 & 100 \\
\hline Loricariidae & 19 & 0,34 & 40 & - & - & \\
\hline \multicolumn{7}{|l|}{ Hypostominae } \\
\hline Liposarcus anisitsi Eigenmann \& Kennedy, 1903 & 1 & 0,02 & 20 & - & - & - \\
\hline Hypostomus sp. & 4 & 0,07 & 20 & - & - & - \\
\hline \multicolumn{7}{|l|}{ Loricariinae } \\
\hline Pseudohemiodon platycephalus Kner, 1854 & 13 & 0,23 & 20 & - & - & - \\
\hline \multicolumn{7}{|l|}{ Hypoptopomatinae } \\
\hline Otocinclus vittatus Regam, 1904 & 1 & 0,02 & 20 & - & - & - \\
\hline Perciformes & 605 & 10,89 & 100 & 462 & 11,30 & 100 \\
\hline Cichlidae & 605 & 10,89 & 100 & 462 & 11,30 & 100 \\
\hline Apistogramma borellii Regan, 1906 & 99 & 1,78 & 100 & 183 & 4,48 & 100 \\
\hline Aequidens plagiozonatus Kullander, 1984 & 18 & 0,32 & 80 & 9 & 0,22 & 80 \\
\hline Laetacara dorsigera Heckel, 1840 & 435 & 7,83 & 100 & 244 & 5,97 & 100 \\
\hline Crenicichla lepidota Heckel, 1840 & 45 & 0,81 & 60 & 22 & 0,54 & 100 \\
\hline Mesonauta festivus Heckel, 1840 & 8 & 0,14 & 20 & 3 & 0,07 & 40 \\
\hline Crenicichla semifasciata Heckel, 1840 & - & - & - & 1 & 0,02 & 20 \\
\hline Gymnotiformes & 5 & 0,09 & 60 & 140 & 3,42 & 100 \\
\hline Gymnotidae & 1 & 0,02 & 20 & 1 & 0,02 & 20 \\
\hline Gymnotus inaequilabiatus Valenciennes, 1839 & 1 & 0,02 & 20 & 1 & 0,02 & 20 \\
\hline Sternopygidae & 1 & 0,02 & 20 & 22 & 0,54 & 60 \\
\hline Sternopygus macrurus Bloch ; Scheneider, 1801 & - & - & - & 1 & 0,02 & 20 \\
\hline Eigenmannia trilineata López \& Castelo, 1966 & 1 & 0,02 & 20 & 21 & 0,51 & 40 \\
\hline Hipopomidae & 3 & 0,05 & 20 & 117 & 2,86 & 100 \\
\hline Brachyhypopomus sp. B & 3 & 0,05 & 20 & 100 & 2,45 & 100 \\
\hline Brachyhyроротиs sp. A & - & - & - & 17 & 0,42 & 40 \\
\hline Cyprinodontiformes & 55 & 0,99 & 80 & 149 & 3,64 & 100 \\
\hline Rivulidae & 55 & 0,99 & 80 & 149 & 3,64 & 100 \\
\hline Rivulus punctatus Boulenger, 1895 & 45 & 0,81 & 80 & 140 & 3,42 & 100 \\
\hline Pterolebias phasianus Costa, 1988 & - & - & - & 4 & 0,10 & 40 \\
\hline Stenolebias damascenoi Costa, 1991 & - & - & - & 2 & 0,05 & 20 \\
\hline Trigonectes balzanii Perugia, 1891 & 9 & 0,16 & 60 & 3 & 0,07 & 20 \\
\hline
\end{tabular}


Tabela 2. Continuação...

\begin{tabular}{|c|c|c|c|c|c|c|}
\hline \multirow[t]{2}{*}{ Ordem/Família/Espécies } & \multicolumn{3}{|c|}{ Caixa } & \multicolumn{3}{|c|}{ Baía } \\
\hline & $\mathbf{N}$ & $\mathbf{N \%}$ & F\% & $\mathbf{N}$ & $\mathbf{N \%}$ & F\% \\
\hline Stenolebias sp. & 1 & 0,02 & 20 & - & - & - \\
\hline Synbranchiformes & 8 & 0,14 & 60 & 21 & 0,51 & 60 \\
\hline Synbranchidae & 8 & 0,14 & 60 & 21 & 0,51 & 60 \\
\hline Synbranchus marmoratus Bloch, 1795 & 8 & 0,14 & 60 & 21 & 0,51 & 60 \\
\hline Lepidosireniformes & - & - & - & 5 & 0,12 & 40 \\
\hline Lepidosirenidae & & & & 5 & 0,12 & 40 \\
\hline Lepidosiren paradoxa Fitzinger, 1837 & - & - & - & 5 & 0,12 & 40 \\
\hline Total & 5557 & & & 4089 & & \\
\hline
\end{tabular}

Tabela 3. Resumo da estrutura da comunidade de peixes (média \pm desvio padrão) e resultado do teste $t$ (T) comparando os locais amostrados no Pantanal de Poconé, MT.

Table 3. Summary of the fish community structure (mean \pm standard deviation) and test $t(\mathrm{~T})$ comparing the sampled sites at Pantanal de Poconé, State of Mato Grosso, Brazil.

\begin{tabular}{lccccc}
\hline \multicolumn{1}{c}{ Variável } & Caixa & Baía & T & DF & P valor \\
\hline Riqueza & $26 \pm 9$ & $25 \pm 6$ & $-0,353$ & 8 & $0,733 \mathrm{~ns}$ \\
Abundância & $1128 \pm 540,6$ & $801 \pm 585,2$ & $-0,920$ & 8 & $0,385 \mathrm{~ns}$ \\
total de peixes & & & & & \\
Biomassa (g) & $362,7 \pm 282,6$ & $225 \pm 42,5$ & $-1,078$ & 8 & $0,313 \mathrm{~ns}$
\end{tabular}

$\mathrm{DF}=$ graus de liberdade, $\mathrm{p}=$ probabilidade, $\mathrm{ns} \mathrm{p}>0,05$ : diferença não significativa.

$\mathrm{DF}=$ degrees of freedom, $\mathrm{p}=$ probability, $\mathrm{ns} \mathrm{p}>0.05$ : no significant difference.

45,7\% foram representadas por 10 ou menos indivíduos. Serrapinnus calliurus foi a espécie dominante nos dois ambientes, com 3193 indivíduos nas caixas de empréstimo, o que corresponde a cerca de $57 \%$ dos espécimes coletados neste ambiente e 2326 indivíduos na baía do Coqueiro, que representaram $56 \%$ do total capturado para este ambiente.

A riqueza, a biomassa e o número total de peixes (g) não variaram entre os ambientes (Tabela 3), e a maioria das espécies foi encontrada em todos os locais amostrados, indicando grande semelhança na composição da ictiofauna, sendo esta comprovada pela análise de similaridade (Anosim), a qual revelou que não existe diferença na composição de espécies entre as caixas de empréstimo e a baía do Coqueiro tanto quantitativamente (abundância) (Anosim R; $-0,218$, $\mathrm{p}=0,946,1000$ permutação), quanto em relação à presença/ausência de espécies (Anosim $\mathrm{R}=-0,09, \mathrm{p}=0,849,1000$ permutações). Tal similaridade na composição de espécies é também mostrada graficamente pelo dendrograma (Figura 4).

\section{Discussão}

A existência de 59 espécies das 269 registradas para o Pantanal por Britski et al. (2007) aponta que esses ambientes apesar de pequenos e alguns de origem artificial, como as caixas de empréstimo, podem ser componentes importantes para a manutenção da ictiofauna local, notadamente no período de cheia. $\mathrm{O}$ potencial de refúgio e a produtividade podem compensar as desvantagens das condições árduas, que podem causar estresse biológico em algumas espécies de peixes (Fisher \& Willis 2000).
Essa riqueza é semelhante à encontrada em outras áreas vegetadas de lagos, em planície de inundação, como em lagoas da parte sul do Pantanal, onde Súarez et al. (2004) registraram 49 espécies, e em lagos na planície de inundação do rio Paraná, onde Petry et al. (2003a) capturam 57 espécies.

A complexidade estrutural e a dinâmica hidrológica da planície de inundação proporcionam vários biótopos que suportam alta biodiversidade, sendo as macróftas aquáticas um dos mais importantes (Lowe-MCconnell 1987, Petry et al. 2003b). Em alguns locais de coleta, a proliferação de macrófitas foi tão grande que cobriu totalmente a superfície da água. Associações entre peixes e macrófitas podem ser explicadas por vários fatores, como a grande disponibilidade de alimento, substrato para desovas e refúgio contra a predação, e, embora macrófitas aquáticas possam ser usadas diretamente como alimento (Agostinho et al. 2003), como para algumas das espécies de ciclídeos capturadas nesse estudo (Machado 2003), a principal contribuição para a cadeia trófica de peixes ocorre na produção de detritos (Araújo-Lima et al. 1986, Benedito-Cecílio et al. 2000).

Segundo Lowe-McConnell (1987), ambientes sazonais tendem a ter diversas comunidades com poucas espécies dominantes. Nos ambientes estudados, a dominância de algumas espécies pode estar relacionada às perturbações sazonais, tais como grandes e imprevisíveis variações no nível da água e oxigênio, o que favorece algumas espécies mais bem adaptadas a essas condições (Jepsen 1997) como, por exemplo, S. calliurus e L. dorsigera.

Characiformes e Siluriformes foram as ordens com o maior número de espécies, constituindo mais da metade da comunidade, o que também tem sido registrado em outros estudos (Meschiatti et al. 2000, Vono \& Barbosa 2001, Granado-Lorencio et al. 2007). No entanto, a abundância de indivíduos de Siluriformes foi pouco representativa, apresentando abundância inferior àquela dos Perciformes. Para o grupo dos Siluriformes, apenas $C$. hastatus teve uma representatividade considerável, ficando entre as cinco espécies mais abundantes, o que pode ser determinado pela elevada transparência da água, considerando que estes tendem a ser mais abundantes em águas turvas (Rodríguez \& Lewis 1994).

Ambientes com alta transparência geralmente possuem maior número de espécies visualmente orientadas (Feyrer et al. 2004, Rodríguez \& Lewis 1997). Nessa categoria podem ser incluídos os ciclídeos (Pouilly \& Rodríguez 2004), que nesse estudo foram bem representativos em termos de abundância de indivíduos, sendo importante componente da assembleia de peixes. Gymotiformes teve maior abundância nos ambientes próximos à baía do Coqueiro, o que provavelmente está relacionado ao comportamento sedentário desses 

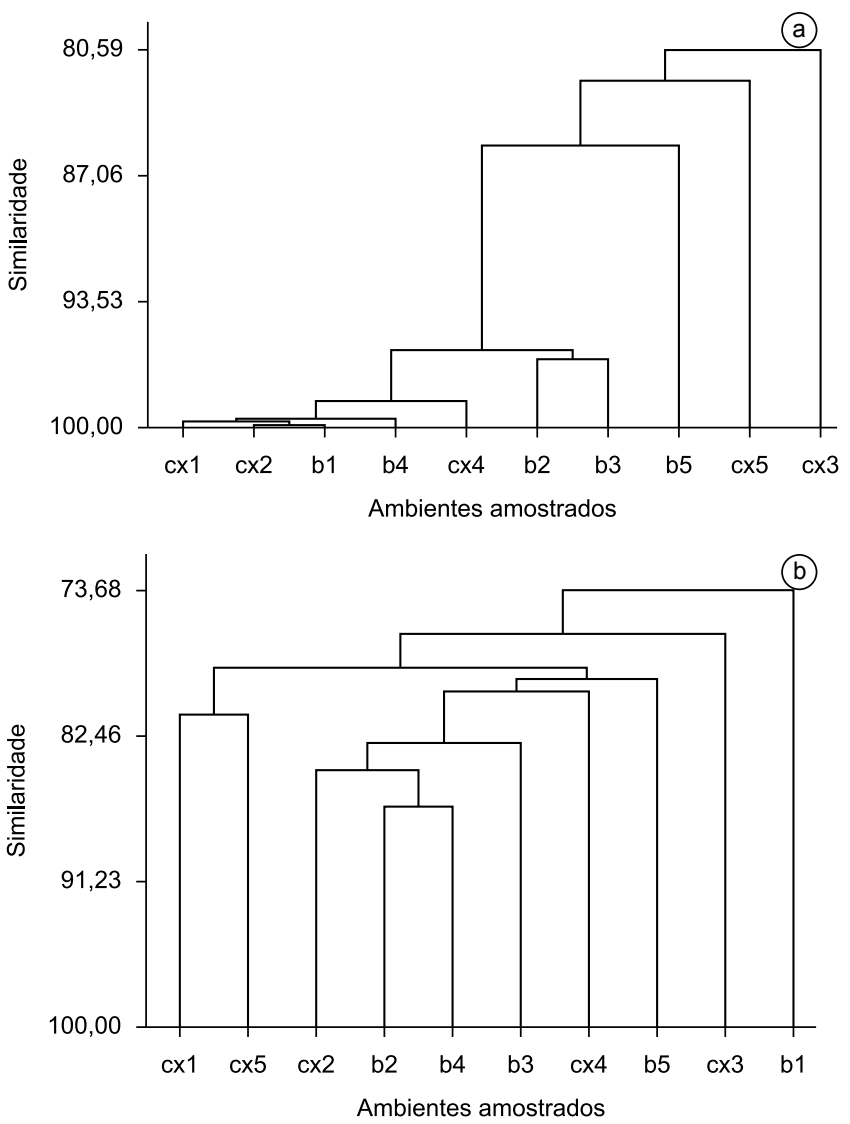

Figura 4. Dendrograma ilustrando a similaridade da ictiofauna. A- obtido por matriz de abundância, $B$ - matriz de presença $\times$ ausência das espécies presentes nas caixas de empréstimo e na baía de Coqueiro, Pantanal de Poconé, MT, Brasil, baseados na distância euclidiana.

Figure 4. Dendrogram illustrating the similarity of the ichthyofauna. A - Obtained by an abundance matrix, B - presence matrix $\times$ absence of species in the loan boxes and in Coqueiro Bay, Pantanal de Poconé, State of Mato Grosso, Brazil, based on Euclidean distances.

indivíduos, ficando assim mais restritos aos pontos próximos a uma das possíveis fontes colonizadoras, a baía do Coqueiro.

As assembléias de peixes dos ambientes amostrados foram predominantemente compostas por espécies de pequeno porte, sendo principalmente representantes da família Characidae; e a dominância de pequenos caracídeos em áreas de água vegetada também tem sido descrita para outros ambientes neotropicais (Cordiviola de Yuan et al. 1984, Meschiatti et al. 2000, Sánchez-Botero \& Araújo-Lima 2001, Súarez et al. 2001, Petry et al. 2003b, Sánchez-Botero et al. 2003, Pelicice et al. 2005, Agostinho et al. 2007). Alguns juvenis de espécies de médio porte, tais como Hoplias malabaricus e Gymnotus inaequilabiatus também foram capturados.

Os ambientes estudados foram caracterizados por baixa abundância de espécies piscívoras, com exceção de Acestrorhinchus pantaneiro e H. malabaricus, nenhum outro piscívoro de médio ou grande porte foi capturado. $H$. malabaricus permanece entre a vegetação de lugares rasos, áreas habitualmente procuradas pelas presas para passarem a noite (Machado 2003), já A. pantaneiro apresentou baixa abundância, o que pode ser atribuído ao fato de que, segundo Machado (2003), essa espécie vive em ambientes rasos, porém raramente em locais vegetados, porque altas densidades de macrófitas podem decrescer a eficiência do predador por reduzir o contato visual com a presa e impedir a movimentação (Priyadarshana et al. 2001).
Em sistemas tropicais, o oxigênio dissolvido é considerado uma importante variável abiótica que afeta a distribuição de peixes e sofre flutuações durante o ciclo diário em regiões litorâneas (Miranda et al. 2000). Estudos indicam que a concentração de oxigênio em áreas vegetadas é reduzida (Junk et al. 1983, Miranda \& Hodges 2000); e de fato, em alguns ambientes a concentração de oxigênio foi baixa, entretanto, as cinco espécies mais abundantes Serrapinnus calliurus, Laetacara dorsigera, Serrapinnus kriegi, Pyrhulina australis, Corydoras hastatus, que representaram $76,21 \%$ dos peixes coletados, não apresentam adaptações respiratórias para tolerar hipoxia. A grande abundância dessas espécies nas áreas de elevada densidade de macrófitas indica que, durante períodos de baixa concentração de oxigênio, a assembleia de peixes possa estar se beneficiando do oxigênio liberado no meio aquático pelo sistema radicular dessas plantas, uma vez que estudos indicam que algumas espécies de macrófitas, inclusive do gênero Eichhornia, o mesmo que predominou nos locais de coleta, podem liberar oxigênio por meio de suas raízes (Moorhead \& Reddy 1988, Jedicke et al. 1989).

Organismos aquáticos que habitam pequenos lagos temporários apresentam uma variedade de adaptações para lidar com estes ambientes. Alguns são generalistas, ocorrendo em diferentes hábitats aquáticos; outros são especialistas e ocorrem apenas em águas temporárias (Bronmark \& Hansson 2005). Formando parte da ictiofauna dos ambientes estudados, encontram-se várias espécies de peixes anuais (Rivulus punctatus, Pterolebias phasianus, Stenolebias damascenoi, Trigonectes balzanii e Stenolebias sp.), todas pertencentes à familia Rivulidae, as quais, de acordo com Costa (2002), ocorrem exclusivamente em corpos de água doce sazonais.

Mudanças no nível da água forçam organismos aquáticos de zonas litorâneas a colonizarem novos hábitats continuamente (Arrington \& Winemiller 2006), então as espécies que se movem com mais agilidade, tais como Ciclídeos e Caracídeos (Willis et al. 2005), que foram bastante abundantes em todos os ambientes amostrados, têm mais chances de colonizar esses ambientes temporários em busca de refúgio contra predadores, proporcionado pela estrutura espacial macrófitas, e de alimento, considerando que as macrófitas servem como substrato para perifiton e invertebrados (Mazzeo et al. 2003, Takeda et al. 2003), os quais fazem parte da dieta de muitas espécies de peixes. Desta forma várias espécies parecem encontrar condições favoráveis nesses hábitats temporários, garantindo sua sobrevivência até atingirem uma fase da vida em que a predação sobre si diminui consideravelmente.

As caixas de empréstimo e a baía do Coqueiro apresentaram alta similaridade na composição de espécies, riqueza, biomassa e número total de peixes. Em planícies de inundação, os ambientes aquáticos tendem a ser mais similares nos períodos chuvosos do que em épocas secas, sendo o grupo de fatores químicos e físicos os primeiros a se tornarem mais similares; depois a composição de zooplâncton, fitoplâncton, peixes e macrófitas vai se tornando cada vez mais similar conforme as chuvas avançam (Thomaz et al. 2007). Assim a diferenciação da fauna em muitos sistemas aquáticos diminui em função da similaridade abiótica (Rahel 2002).

Nos ambientes estudados a condutividade, profundidade, oxigênio dissolvido e a transparência quase não variaram, apenas os valores de $\mathrm{pH}$, temperatura e biomassa de macrófita apresentaram variação entre os ambientes. No entanto, a ordem de magnitude de variação do pH e temperatura foi pequena. Com relação à biomassa de macrófitas, a variação foi maior, uma vez que as espécies dominantes diferiam entre as caixas de empréstimo e a baía do Coqueiro. Mesmo hábitats com características ambientais similares, tais como corpos de água temporários, podem ter faunas distintas por causa das diferentes probabilidades de colonização e extinção das espécies (Pazin et al. 
2006). As espécies que colonizam as águas temporárias apresentam eficientes meios de dispersão (Bronmark \& Hansson 2005), e em planícies alagáveis, no período chuvoso, como resultado da inundação de extensas áreas, os peixes possuem um enorme ambiente para se movimentar (Saint-Paul et al. 2000); desse modo, muitas espécies têm chance de se dispersar pelo sistema, aumentando a probabilidade de colonização.

Nesse contexto, a similaridade na composição das assembléias de peixes entre os ambientes estudados deve-se a fatores diretamente relacionados ao período de inundação, que é a homogeneização do ambiente, e aos movimentos migratórios dentro da planície. Esses fatores permitem que as espécies se distribuam entre os corpos de água da planície, o que aumenta a probabilidade das espécies raras se dispersarem e colonizarem novos hábitats, fato de suma importância, considerando que muitas espécies necessitam de lugares específicos em determinados estágios (Thomaz et al. 2007). Consequentemente a conectividade hidrológica é um importante fator a ser considerado para manutenção da diversidade biológica, sendo que a interrupção da conexão entre os ambientes da planície pode ter efeitos negativos para a biodiversidade a longo e médio prazos, por eliminar oportunidades dos organismos de colonizarem hábitats importantes para completarem seus ciclos de vida (Thomaz et al. 2007, Henning et al. 2007). Isso demonstra forte efeito do pulso de inundação na assembleia de peixes em planície de inundação, onde este é a principal força responsável pela existência, produtividade e interação entre as espécies em um sistema rio-planíce de inundação (Junk et al. 1989).

Muitas espécies de peixes são bastante sensíveis a ligeiras alterações da qualidade da água ou à perda da cobertura vegetal circundante, outras possuem área de distribuição reduzida, o que agrava ainda mais a situação (Granado-Lorencio et al. 2007). Os Brejos e lagoas temporárias têm sido drasticamente destruídos, tanto em áreas agropecuárias como em áreas em processo de urbanização, por meio de drenagem, aterros e desmatamento (Eggleton et al. 2005). E muitas vezes esses processos resultam na perda da conectividade, o que limita as migrações sazonais de organismos aquáticos, reduzindo a diversidade e produtividade de hábitats aquáticos (Rahel 2007, Robinson et al. 2002). A compreensão das conseqüências da inundação, no que diz respeito à dispersão, adaptação e sucessão ecológica das espécies, é de grande relevância para o desenvolvimento de estratégias eficientes para a conservação e manejo (Ward \& Tockner 2001), considerando que mudanças ao longo do tempo no pulso de inundação podem resultar em fundamentais alterações ecológicas nas áreas afetadas (Junk \& Cunha 2005). Assim, estudos de longo prazo e com mais réplicas espaciais e temporais poderiam gerar informações que melhor explicariam a dinâmica que opera nessa planície de inundação, visto que a fauna de peixe que habita esses ambientes é altamente vulnerável aos vários tipos de impactos que os afeta diretamente.

\section{Agradecimentos}

Agradecemos a CAPES pela concessão da bolsa de estudos para o primeiro autor. Ao Dr. Cesar Enrique de Melo que contribuiu muito para a melhoria do manuscrito. Ao Izaias Médice Fernandes pelo auxílio nas análises estatísticas. Aos revisores pelas críticas e sugestões.

\section{Referências Bibliográficas}

ARAÚJO-LIMA, C.A.R.M., FORSBERG, B.R., VICTÓRIA, R.L. \& MARTINELLI, L.A. 1986. Energy sources for detritivorous fi shes in the Amazon. Science 234:256-1258.
AGOSTINHO, A.A., GOMES, L.C. \& FERREIRA, H.J. 2003. Relações entre macrófitas aquáticas e fauna de peixes. In Ecologia e manejo de macrófitas aquáticas (S.M. Thomaz \& L.M. Bini, eds). EDUEM, Maringá, p. 261-279.

AGOSTINHO, A.A., THOMAZ, S.M., GOMES, L.C. \& BALTAZAR, S.L.S.M.A. 2007. Influence of the macrophyte Eichornia azurea on fish assemblage of the upper Paraná River flooplain (Brazil). Aquat. Ecol. 41:619-641.

ARRINGTON, D.A. \& WINEMILLER, K.O. 2006. Habitat affinity, the seasonal flood pulse, and community assembly in the littoral zone of a Neotropical floodplain river. J. N. Am. Benthol. Soc. 25:126-141.

BENEDITO-CECÍLIO, E., ARAUJO-LIMA, C.A.R.M., FORSBERG, B.R., BITTENCOURT, M.M. \& MARTINELLI, L.C. 2000. Carbon source of Amazonian fisheries. Fisheries Manag. Ecol. 7:305-315.

BRITSKI, H.A., SILIMON, K.Z.S. \& LOPES, B.S. 2007. Peixes do Pantanal: manual de identificação. 2 ed. EMPRAPA, Brasília.

BRONMARK, B. \& HANSSON, L.A. 2005. The Biology of Lakes and Ponds. 2 ed. Oxford University Press, New York.

CLARKE, K.R. 1993. Non-parametric multivariate analyses of changes in communitys structure. Aust. J. Ecol. 18:117-143.

COLLINSON, N.H., BIGGS, J., CORFIELD, A., HODSON, M.J., WALKER, D., WHITFIELD, M. \& WILLIAMS, P.J. 1995. Temporary and permanent ponds: an assessment of the effects of drying out on the conservation value of aquatic macroinvertebrate communities. Biol. Conserv. 74:125-133.

COSTA, W.J.M. 2002. Peixes anuais brasileiros: diversidade e conservação. Ed. da UFPR, Curitiba.

CORDIVIOLA DE YUAN, E., OLDANI, N., OLIVEROS, O. \& PIGNALBERI DE HASSAN, C. 1984. Aspectos limnologicos de ambientes próximos la cuidade de Santa Fe (Paraná Medio): poblaciones de peces ligadas a La vegetacion. Neotropica 30(84):127-139.

EGGLETON, M.A., RAMIREZA, R., HARGRAVEA, C.W., GIDOA, K.B., MASONERE, J.R., SCHNELLA, G.D. \& MATTHEWSA, W.J. 2005. Predictability of littoral-zone fish communities through ontogeny in Lake Texoma, Oklahoma-Texas, USA. Environ. Biol. Fish. 73:21-36.

FEYRER, F., SOMMER, T.R., ZEUG, S.C., LEARY, G.O. \& HARRELL, W. 2004. Fish assemblages of perennial floodplain ponds of the Sacramento River, California (USA), with implications for the conservation of native fishes. Fisheries Manag. Ecol. 11:335-344.

FISHER, S.J. \& WILLIS, D.W. 2000. Seasonal dynamics of aquatic fauna and habitat parameters in a perched upper Missouri river wetland. Wetlands 20(3):470-478.

GRANADO-LORENCIO, C., LOBÓN-CERVIÁ, J. \& ARAUJO LIMA, C.R.M. 2007. Floodplain lake fish assemblages in the Amazon River: directions in conservation biology. Biodivers. Conserv. 16:679-692.

HAMILTON, S.K., SIPPEL, S.J. \& MELACK, J.M. 2002. Comparison of inundation patterns among major South American floodplains. J. Geophys. Res. 107:31-44.

HENNING, J.A., GRESSWELL, R.E. \& FLEMING, I.A. 2007. Use of seasonal freshwater wetlands by fishes in a temperate river floodplain. J. Fish Biol. 71:476-492.

JACKSON, D.A., PERES-NETO, P.R. \& OLDEN, J.D. 2001. What controls who is where in freshwater fish communities: the roles of biotic, abiotic, and spatial factors. Can. J. Fish. Aquat. Sci. 58:157-170.

JEDICKE, A.B., SAINT-PAUL, F.U. \& SCHLUTER, U.B. 1989. Increase in the oxygen concentration in Amazon waters resulting from the root exudation of two notorious water plants, Eichhornia crassipes (Pontederiaceae) and Pistia stratiotes (Araceae). Amazoniana 11:53-69.

JEPSEN, D.B. 1997. Fish species diversity in sand bank habitats of a neotropical river. Environ. Biol. Fish. 49:449-460.

JUNK, W.J., BAYLEY, P.B. \& SPARKS, R.S. 1989. The flood pulse concept in river - floodplain systems. Can. Spec. Publ. Fish. Aquat Sci.106:110-127.

JUNK, W.J., SOARES, G.M. \& CARVALHO, F.M. 1983. Distribution of fish species in a lake of the Amazon River floodplain near Manaus 
(lago Camaleão), with special reference to extreme oxygen conditions. Amazoniana 7:397-431.

JUNK, W.J. \& CUNHA, C.N.D. 2005. Pantanal: a large South American wetland at a crossroads. Ecol. Eng. 24:391-401.

JUNK, W.J., CUNHA, C.N., WANTZEN, K.M., PETERMANN, P., STRÜSSMANN, C., MARQUES, M.I. \& ADIS, J. 2006. Biodiversity and its conservation in the Pantanal of Mato Grosso, Brazil. Aquat. Sci. 68:278-309.

LOWE-MCCONNELL, R.H. 1987. Ecological studies in tropical fish communities. Cambridge University Press, England.

MACHADO, F.A. 2003. História natural de peixes do Pantanal: com destaque em hábitos alimentares e defesa contra predadores. Tese de doutorado, Universidade de Campinas, São Paulo.

MAGURRAN, A.E. 2004. Measuring biological diversity. Blackwell Publishing, Oxford.

MAZZEO, N., RODRÍGUEZ-GALEGO, L. \& KRUK, C. 2003. Effects of Egeria densa Planch. beds on a shallow lake without piscivores fish. Hydrobiologia 506/509:591-602.

MESCHIATTI, A.J., ARCIFA, M.S. \& FENERICH-VERANI, N. 2000. Fish communities associated with macrophytes in Brazilian floodplain lakes. Environ. Biol. Fish. 58:133-143.

MIRANDA, L.E. \& HODGES, K.B. 2000. Role of aquatic vegetation coverage on hypoxia and sunfish abundance in bays of a eutrophic reservoir. Hydrobiologia 427:51-57.

MIRANDA, L.E., DRISCOLL, M.P. \& ALLEN, M.S. 2000. Transient physicochemical microhabitats facilitate fish survival in inhospitable aquatic plant stands. Freshwater Biol. 44:617-628.

MOORHEAD, K.K. \& REDDY, K.R.R. 1988. Oxygen transport through selected aquatic macrophytes. J. Environ. Qual. 17:138-142.

MORAN-LOPEZ, R., PEREZ-BOTE, J.L., SILVA, R.E. \& CORBACHO, A.C. 2005. Summer habitat relationships of barbells in south-west Spain. J. Fish Biol. 67:66-82.

OLIVEIRA, A.C.B., SOARES, G.M.M., MARTINELLI, L.A. \& ZACARIAS, M.M. 2006. Carbon sources of fi sh in an Amazonian floodplain lake. Aquat. Sci. 68:229-238.

PAZIN, V.F.V., MAGNUSSON, W.E., ZUANON, J. \& MENDONÇA, F.P. 2006. Fish assemblages in temporary ponds adjacent to 'terra firme' streams in Central Amazonia. Freshwater Biol. 51:1025-1037.

PELICICE, F.M., AGOSTINHO, A.A. \& THOMAZ, S.M. 2005. Fish assemblages associated with Egeria in a tropical reservoir: investigating the effects of plant biomass and diel period. Acta Oecol. 27:9-16.

PETRY, A.C., AGOSTINHO, A.A. \& GOMES, L.C. 2003a. Fish assemblages of tropical floodplain lagoons: exploring the role of connectivity in a dry year. Neotrop. Ichthyol. 1(2):111-119.

PETRY, P., BAYLEY, P.B. \& MARKLE, D.F. 2003b, Relationships between fish assemblages, macrophytes and environmental gradients in the Amazon River Floodplain. J. Fish Biol. 63:547-579.

POUILLY, M. \& RODRÍGUEZ, M.A. 2004. Determinism of fish assemblage structure in neotropical floodplain lakes: influence of internal and landscape lake conditions. In Proceedings of the Second International Symposium on the management of Large Rivers for Fisheries (R. Welcomme \& T. Petr, eds). FAO, Bangkok, p.243-265.

PRIYADARSHANA, T., ASAEDA, T. \& MANATUNGE J. 2001. Foraging behaviour of planktivorous fish in artificial vegetation: the effects on swimming and feeding. Hydrobiologia 442:231-239.

$\mathrm{R}$ development core team. 2005. R: a language and environment for statistical computing. R Foundation for Statistical Computing, Vienna. disponível em www.r-project.org
RAHEL, F.J. 2002. Homogenization of freshwater faunas. Annu. Rev. Ecol. Syst. 33:291-315

RAHEL, F.J. 2007. Biogeographic barriers, connectivity and homogenization of freshwater faunas: it's a small world after all. Freshwater Biol. 52:696-710

RICKLEFS, R.E. 1987. Community diversity: relative roles of local and regional processes. Science 235:167-171.

ROBINSON, C.T., TOCKNER, K. \& WARD, J.V. 2002. The fauna of dynamic riverine landscapes. Freshwater Biol. 47:661-677.

RODRÍGUEZ, M.A. \& LEWIS, W.M. 1994. Regulation and stability in fish assemblages of neotropical floodplain lakes. Oecologia 99:166-180.

RODRÍGUEZ, M.A. \& LEWIS, W.M. 1997. Structure of fish assemblages along environmental gradients in floodplain lakes of the Orinoco river. Ecol. Monogr. 67(1):109-128.

SAINT-PAUL, U., ZUANON, J., CORREA, M.A.V., GARCIA, M., FABRE, N.N., BERGER, U. \& JUNK, W.J. 2000. Fish communities in central Amazonian white and black water floodplains. Environ. Biol. Fish. 57:235-250.

SÁNCHEZ-BOTERO, J.L. \& ARAÚJO-LIMA, C.A.R.M. 2001. As macrófitas aquáticas como berçário para a ictiofauna da várzea do rio Amazonas. Acta amazon. 31(3):437-447.

SÁNCHEZ-BOTERO, J.I.S., FARIAS, M.L., PIEDADE, M.T., GARCEZ, D.S. 2003. Ictiofauna associada às macrófitas aquáticas Eichhornia azurea (SW.) Kunth. e Eichhornia crassipes (Mart.) Solms. no lago Camaleão, Amazônia Central, Brasil. Acta Sci., Biol. Sci. 25(2):369-375.

SÚAREZ, Y.R., PETRERE Jr., M. \& CATELLA, A.C. 2001. Factors determining the structure of fish comunities in Pantanal lagoons (MS, Brazil). Fisheries Manag. Ecol. 8:173-186.

SÚAREZ, Y.R., PETRERE-JR, M. \& CATELLA, A.C. 2004. Factors regulating diversity and abundance of fish communities in Pantanal lagoons, Brazil. Fisheries Manag. Ecol. 11:45-50.

Systat. 2004. Systat for Windows. Versão 11. Systat Software Inc., Chicago.

TAKEDA, A.M., SOUZA-FRANCO, G.M., MELO, S.M. \& MONKOLSKI, A. 2003. Invertebrados associados às macrófitas aquáticas da planície de inundação do alto rio Paraná (Brasil). In Ecologia e Manejo de Macrófitas Aquáticas (S.M.Thomaz \& L.M. Bini, eds). EDUEM, Maringá, p. 243-260.

THOMAZ, S.M., BINI, L.M. \& BOZELLI, R.L. 2007. Floods increase similarity among aquatic habitats in river-floodplain systems. Hydrobiologia 579:1-13.

VONO, V. \& BARBOSA, F.A.R. 2001. Habitats and littoral zone fish community structure of two natural lakes in southeast Brazil. Environ. Biol. Fish. 61:371-379.

WANTZEN, K.M., MACHADO, F.A., VOSS, M., BORISS, H. \& JUNK, W.J. 2002. Seasonal isotopic shifts in fish of the Pantanal wetland. Brazil Aquat. Sci. 64:239-251.

WARD, J.V. \& TOCKNER, K. 2001. Biodiversity: towards a unifying theme for river ecology. Freshwater Biol. 46:807-819.

WILLIS, S.C., WINEMILLER, K.O. \& LOPEZ-FERNANDEZ, H. 2005. Habitat structural complexity and morphological diversity of fish assemblages in a neotropical floodplain river. Oecologia 142:284-295.

Recebido em 16/11/09 Versão reformulada recebida em 10/05/10 Publicado em 20/05/10 